Document downloaded from:

http://hdl.handle.net/10251/58590

This paper must be cited as:

Gómez García, S.; Naranjo Ornedo, V.; Miralles Ricós, R. (2011). Removing interference components in time frequency representations using morphological operators. Journal of Visual Communication and Image Representation. 22(1):401-410. doi:10.1016/j.jvcir.2011.03.007.

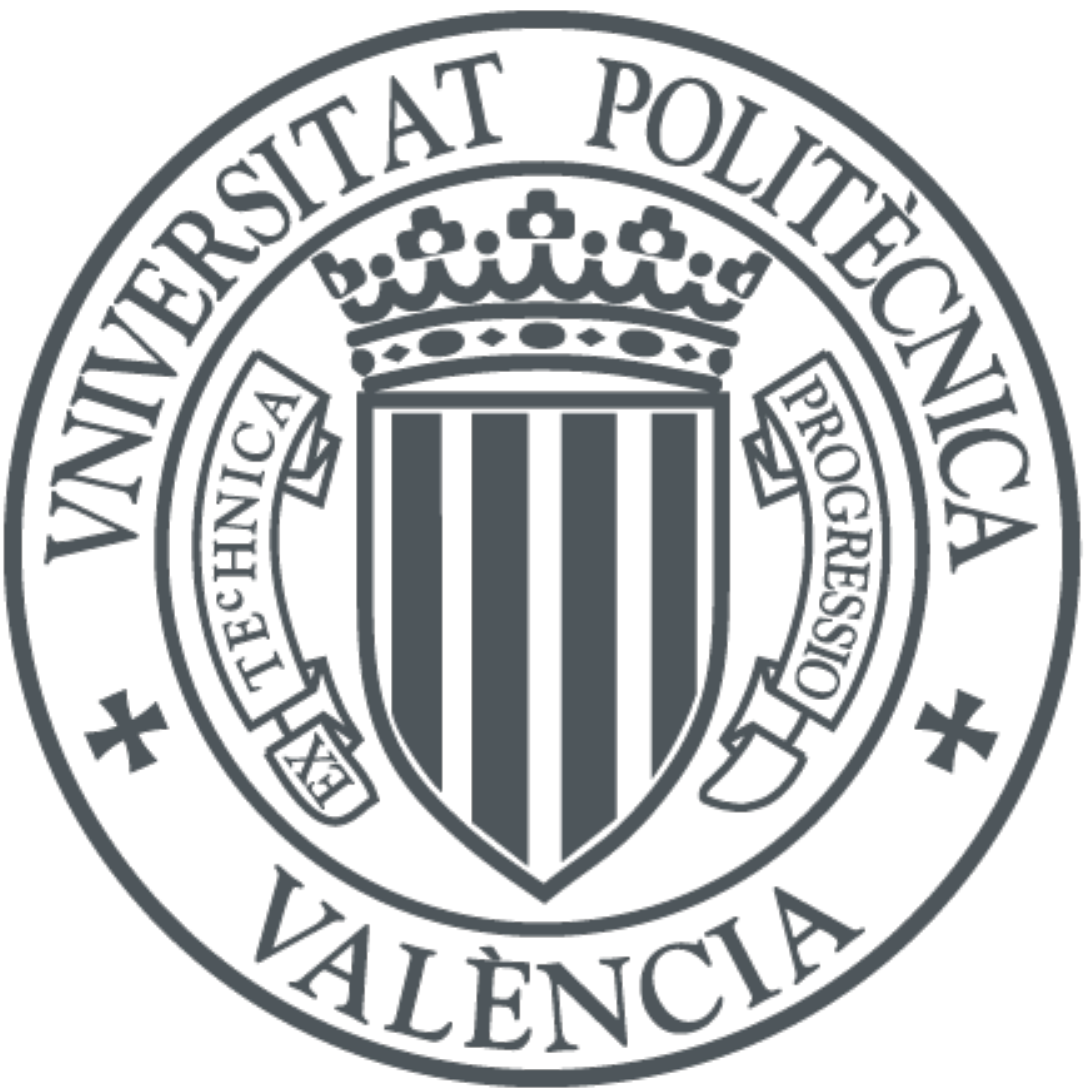

The final publication is available at

http://dx.doi.org/10.1016/j.jvcir.2011.03.007

Copyright Elsevier

Additional Information 


\title{
Removing interference components in time-frequency representations using morphological operators
}

\author{
Soledad Gómez ${ }^{\mathrm{a}, 1}$, Valery Naranjo ${ }^{\mathrm{b}}$, Ramón Miralles,a,1 \\ ${ }^{a}$ Instituto de Telecomunicaciones y Aplicaciones Multimedia (iTEAM), Dpto. de \\ Comunicaciones, Universidad Politécnica de Valencia, Camino de Vera S/N, 46022, \\ Valencia, Spain \\ ${ }^{b}$ Instituto de Bioingeniería y Tecnología orientada al Ser Humano, Universidad Politécnica \\ de Valencia, Camino de Vera S/N, 46022, Valencia, Spain
}

\begin{abstract}
Time-frequency representations have been of great interest in the analysis and classification of non-stationary signals. The use of highly selective transformation techniques is a valuable tool for obtaining accurate information for studies of this type. The Wigner-Ville distribution has high time and frequency selectivity in addition to meeting some interesting mathematical properties. However, due to the bi-linearity of the transform, interference terms emerge when the transform is applied over multi-component signals. In this paper, we propose a technique to remove cross-components from the Wigner-Ville transform using image processing algorithms. The proposed method exploits the advantages of non-linear morphological filters, using a spectrogram to obtain an adequate marker for the morphological processing of the Wigner-Ville transform. Unlike traditional smoothing techniques, this algorithm provides crossterm attenuations while preserving time-frequency resolutions. Moreover, it could also be applied to distributions with different interference geometries. The method has been applied to a set of different time-frequency transforms, with promising results.
\end{abstract}

Key words: Time-frequency representations, morphological image processing.

\section{Introduction}

Time-frequency representations $[1,2]$ are essential when working with nonstationary signals such as ultrasonic or acoustic signals. The most straightforward technique for representing a signal in the time-frequency plane is the

\footnotetext{
*Corresponding author: sogogar@upvnet.upv.es

Email addresses: sogogar@upvnet.upv.es (Soledad Gómez), vnaranjo@dcom.upv.es (Valery Naranjo), rmiralle@dcom.upv.es (Ramón Miralles)

${ }^{1}$ This work was supported by the national R+D program under grant TEC2008-02975 (Spain), FEDER programme and Generalitat Valenciana CMAP 340.
} 
short time Fourier transform (STFT). This technique consists of prewindowing the signal under test $x(t)$ around a particular time $t$ and calculating its Fourier transform. The squared modulus of the STFT, the spectrogram, is commonly used for the analysis of non-stationary signals in the time-frequency domain [3]. Nevertheless, as a result of the Heisenberg-Gabor principle [4], the spectrogram has a trade-off between time and frequency resolutions. When using the spectrogram, the results are dependent on the choice of the window length, which limits the number of applications where this can be applied.

Other types of time-frequency representations are based on the Wigner-Ville distribution (WVD). The WVD is defined as:

$$
W_{x}(t, \nu)=\int_{-\infty}^{\infty} x(t+u / 2) x^{*}(t-u / 2) e^{-j 2 \pi \nu u} d u
$$

The WVD satisfies some interesting mathematical properties $[5,2,6]$, such as marginal properties or preservation of time and frequency shifts. Moreover, the WVD simultaneously provides high selectivity in time and frequency while the spectrogram has a trade-off between time and frequency resolutions. Despite this, the WVD has a drawback because it produces a great number of crosscomponents when it is applied over multi-component signals. Taking the above into consideration, the main objective of this study is to achieve a time-frequency representation, without cross-terms (such as the spectrogram), which can be as selective as possible in time-frequency planes (such as the WVD).

This paper presents an innovative method for the elimination of cross-components in the WVD. In addition to cross-term attenuation, our algorithm preserves time-frequency resolutions. To do this, it incorporates image-processing algorithms based on mathematical morphology.

A review of different techniques for the reduction of cross-components from the WVD is presented in Section 2. The basis to understand the presented research and the proposed method, which is based on morphological filters, are explained in Sections 3 and 4, respectively. Finally, some results and conclusions are discussed in Sections 5 and 6.

\section{State of The Art}

The interference structure of the WVD and its properties have been the subject of extensive studies $[6,7]$. Research on eliminating cross-components from the WVD has been of enormous benefit when working with multi-component signals. Since interference terms are oscillatory, previous studies were related to attenuate interference components by means of a smoothing operation. These studies lie in the convolution of the WVD with a 2-D smoothing kernel $[7,8]$. The Cohen class of shift-invariant transformations provides an important framework for WVD smoothing although the design of a satisfactory kernel is a complex task. Many research papers have been published on this topic with the objective of achieving cross-term attenuation in the WVD, while preserving time-frequency resolutions and all the desired mathematical properties. As an 
example, we can refer to [9], where the most suitable kernel is selected by means of processing the corresponding image of the ambiguity function. Some other proposals related to kernel design can be seen in $[10,11]$. The main difficulty of these techniques is the selection of the most appropriate kernel for each type of signal.

The approach presented in [12] reduces the cross-components by combining the Fourier-Bessel expansion with the WVD. The Fourier-Bessel expansion decomposes a multi-component signal into a number of mono-component signals, and the WVD is applied over each mono-component signal. It is a powerful tool to eliminate interferences from the WVD. However, the method has one restriction - it assumes that signal components are well-separated in the frequency domain. If the signal components are not well-separated, it is likely that the method will not correctly perform the frequency components separation, as will be discussed in Section 5. An improvement of this method is presented in [13]. This improvement will be also tested and compared with our method in Section 5 .

Alternative approaches to remove cross-components from the WVD distribution are based on the use of image-processing algorithms [14, 15]. However, these methods assume a-priori knowledge of the interference geometry.

A new method to remove interferences from the WVD is presented in this paper. It starts from our earlier contribution [16] and represents an improvement on the obtained results. We overcome the main drawback of [16] since that method was not appropriate to reduce interferences when cross-terms are touching signal terms. In this paper, we introduce another morphological operator to remove interferences from the WVD even when interferences overlap signal terms. New simulations have also been carried out and discussed to demonstrate the application of the proposed method not only to remove interferences from the WVD but also to remove them from distributions with different interference geometries. The results from $[12,13]$ are compared to those obtained using our method.

\section{Method Basis}

The basic idea of the presented method is to apply morphological filters to reduce interference components (cross-terms) from images corresponding to noisy time-frequency representations. We want to recover only those signal terms from the WVD that are also present in the spectrogram distribution, thereby preserving the resolution of the WVD. The method uses the image corresponding to the spectrogram as a marker for a morphological reconstruction carried out over the WVD, which we are seeking to denoise. Essentially, the method uses the opening by $\lambda$-reconstruction operator (or for simplicity $\lambda$-reconstruction), which is described below.

\subsection{Definition of $\lambda$-reconstruction}

The $\lambda$-reconstruction operator belongs to a family of transformations called geodesic operators $[17,18,19,20]$. A geodesic operator involves two input im- 
ages: the marker and the reference. A morphological transformation is applied to the marker and the result is forced to remain above or below the reference. Let $f$ and $g$ (two grayscale images) be the reference and the marker images, respectively, which are both defined in the same domain as:

$$
f(\mathbf{x}): \mathrm{E} \rightarrow \mathcal{T},
$$

where $(\mathbf{x}) \in \mathrm{E}$ is the pixel position. In the case of valued discrete images, $\mathcal{T}=\left\{t_{\text {min }}, t_{\text {min }}+1, \ldots t_{\text {max }}\right\}$ (in general $\mathcal{T} \subset \mathbb{Z}$ or $\mathbb{R}$, or any compact subset of $\mathbb{Z}$ or $\mathbb{R}$ ) is an ordered set of grey-levels. Typically, in digital 8-bit images we have $t_{\text {min }}=0$ and $t_{\text {max }}=255$.

Let us define the unitary geodesic $\lambda$-dilation of the marker $g$ with respect to the reference $f, \delta_{f, \lambda}^{(1)}(g)$, as the point-wise minimum between the reference and the unitary non-flat dilation, $\delta_{\lambda}^{(1)}$, of the marker, which is:

$$
\delta_{f, \lambda}^{(1)}(g)=\delta_{\lambda}^{(1)}(g(\mathbf{x})) \wedge f(\mathbf{x}) .
$$

The unitary $\lambda$-dilation, $\delta_{\lambda}^{(1)}$, represents the dilation with a unitary nonflat structuring function $b(\mathbf{x})$, with $b(\mathbf{x}) \in \mathcal{F}\{\mathrm{E}, \mathcal{T}\}$ being a weighting function defined as:

$$
b(\mathbf{x})= \begin{cases}-\lambda & \mathbf{x} \in B \\ -\infty & \mathbf{x} \notin B\end{cases}
$$

Thus, the unitary $\lambda$-dilation, $\delta_{\lambda}^{(1)}$, will be defined by the expression:

$$
\delta_{\lambda}^{(1)}(f)(\mathbf{x})=\left\{f(\mathbf{y}): f(\mathbf{y})=\sup [f(\mathbf{z})-\lambda], \mathbf{z} \in B_{\mathbf{x}}\right\} \vee f(\mathbf{x}) .
$$

The geodesic $\lambda$-dilation of size $n$ of the marker $g$ with respect to the reference $f$ is obtained by performing $n$ successive geodesic $\lambda$-dilations of $g$ with respect to $f$ :

$$
\delta_{\lambda, f}^{(n)}(g)=\delta_{f, \lambda}^{(1)}\left[\delta_{f, \lambda}^{(n-1)}(g)\right]
$$

with $\delta_{f, \lambda}^{(0)}(g)=g$.

The $\lambda$-reconstruction [21] of the reference image $f$ from the marker $g$ is defined as the geodesic $\lambda$-dilation of $g$ with respect to $f$ until stability:

$$
\gamma_{\lambda}^{r e c}(f(\mathbf{x}), g(\mathbf{x}))=\delta_{f, \lambda}^{(k)}(g(\mathbf{x})),
$$

where $k$ is $\delta_{f, \lambda}^{(k)}(g)=\delta_{f, \lambda}^{(k+1)}(g)$.

Using the $\lambda$-reconstruction operator, only the reference image pixels that actually touch the marker will be reconstructed with the maximum value of the reference. The remainder of the connected areas touched by the marker will be reconstructed with an intensity level that will decrease with a slope equal to $\lambda$ $[22,21]$. 


\subsection{Use and behaviour of $\lambda$-reconstruction}

The results of applying the $\lambda$-reconstruction operator are explained in Figures 1 and 2 using a simulated signal to illustrate the performance of the operator. Test images have been used to illustrate the example. These images are composed by straight lines and zigzag lines. We consider the zigzag lines to be the undesired lines and the straight lines those to be preserved. This example demonstrates how we are able to reduce zigzag lines using the $\lambda$-reconstruction operator, and how, when the undesired lines overlap the desired ones, the use of different values of $\lambda$ provides satisfactorily differentiated results.

In Figure 1, the undesired zigzag lines do not overlap the desired straight lines. In contrast, in Figure 2, the undesired zigzag lines overlap the desired straight lines.

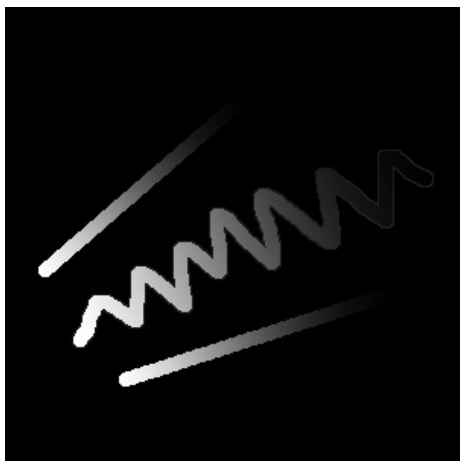

(a)

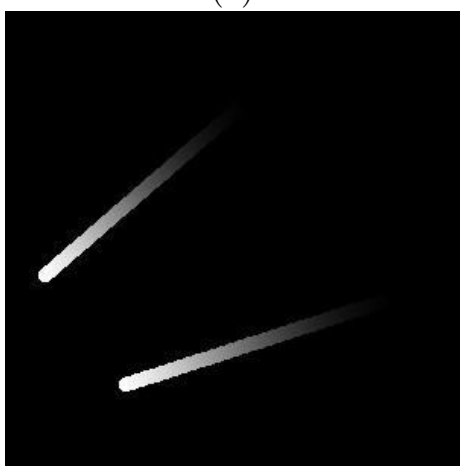

(c)

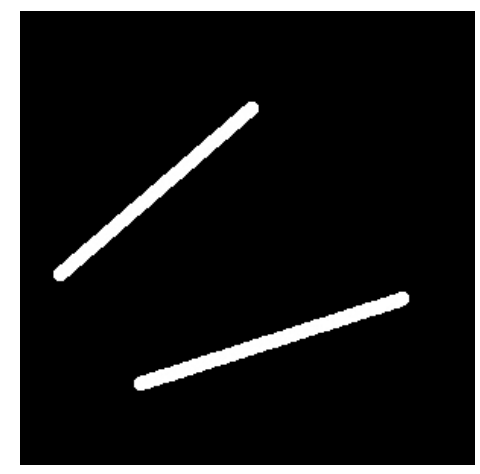

(b)

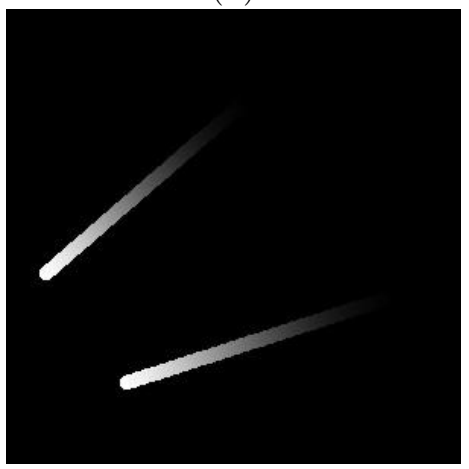

(d)

Figure 1: (a) Reference image. (b) Marker. (c) Result of $\lambda$-reconstruction with $\lambda=0$. (d) Result of $\lambda$-reconstruction with $\lambda=15$.

Focussing on Figure 1, our aim is to prove how the operator is able to reconstruct only the straight lines (desired lines). Image 1.b is used as marker for the morphological operation carried out over Image 1.a. By using the $\lambda$ reconstruction operator with different values of $\lambda(\lambda=0$ and $\lambda=15)$, we obtain images 1.c and 1.d, respectively. From here, we can point out how the $\lambda$ - 


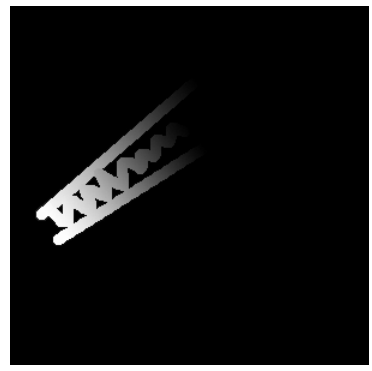

(a)

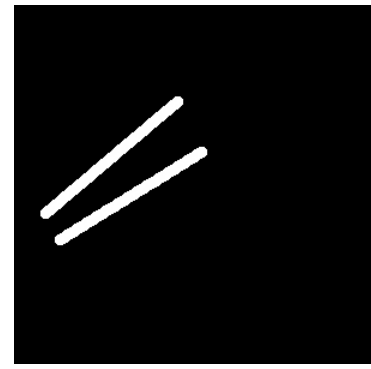

(b)

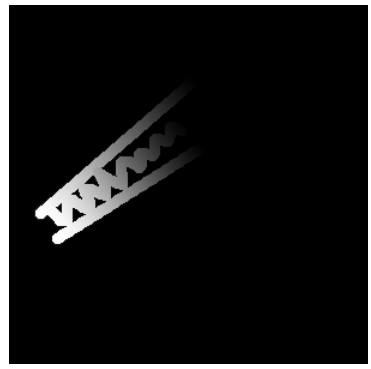

(c)

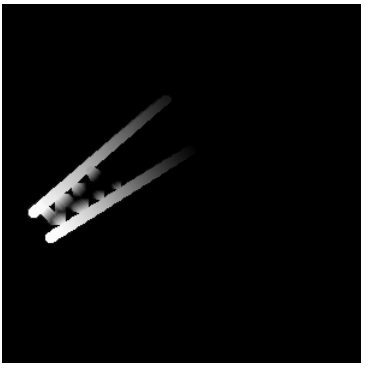

(d)

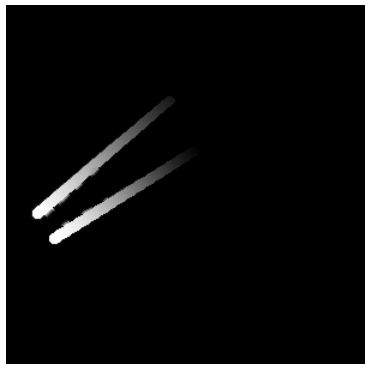

(e)

Figure 2: (a) Reference image. (b) Marker. (c) Result of $\lambda$-reconstruction with $\lambda=0$. (d) Result of $\lambda$-reconstruction with $\lambda=15$. (e) Result of $\lambda$-reconstruction with $\lambda=30$.

reconstruction operation provides identical solutions independently of the value of $\lambda$ and efficiently removes the undesired lines. Note that in this example the zigzag lines do not overlap the desired ones.

Focussing on Figure 2, our aim is also to reconstruct only the straight lines. Note how, in this case, the undesired zigzag lines overlap the desired straight lines. Image 2.b is used as marker for the morphological operation carried out over the reference image 2.a. By using the $\lambda$-reconstruction operator with values of $\lambda=0, \lambda=15$, and $\lambda=30$, we obtain images 2.c, 2.d, and 2.e, respectively. Note that if a value of $\lambda=0$ is used, the operator does not provide good results because the zigzag lines that touch the marker have also been reconstructed. Higher values of $\lambda$ provide a good solution for removing these undesired components. Note that if $\lambda$ increases, the intensity of the interferences decreases.

By considering these two examples, we can draw the following conclusions: when signals and cross-components are not touching, the operator achieves satisfactory results independent of the value of $\lambda$. However, when the signal and cross-terms are touching, the $\lambda$-reconstruction operator still achieves satisfactory results, but the selection of an appropriate value of $\lambda$ will improve the result. The selection of the correct marker will also improve the final results as will be justified in the next section.

The morphological reconstruction operator was presented in [16] as a useful tool to reduce cross-components from interfered diagrams. The method pro- 
posed in [16] performs satisfactory solutions only when cross-terms do not touch signal terms. The standard reconstruction operator, as introduced in [16], represents a particular case of the $\lambda$-reconstruction operator for values of $\lambda=0$.

In the next section, a general scheme describing the complete method is presented. The method has been called $\lambda$-Reconstruction Cross-Component Removal ( $\lambda$-RCCR).

\section{General scheme of $\lambda$-RCCR}

The basis of the $\lambda$-RCCR method is outlined in Figure 3. The central idea of the method is to recover the signal information from those time-frequency representations suffering from interference terms. The method performs a morphological reconstruction operation. A binary image is obtained from the spectrogram, and it is used as a marker in order to ensure the inclusion of only signal terms in the marker. To improve accuracy, the marker must be pre-processed. For example, in those applications where time and frequency resolutions are simultaneously required, the method should be applied using the skeleton of the spectrogram as a marker and the image corresponding to a WVD as a reference.

The skeleton of the spectrogram is obtained by performing the homotopic skeleton associated to the iteration of the thinning transformation [17, 20]. This algorithm is based on the Hit-or-miss transform, which removes points of a binary set according to a finite family of templates but preserving the homotopy of the set. Note also that the approach does not require any parameter. This skeleton is a signal that contains the minimum quantity of information that is sufficient to locate each point of the support of the signal in frequency and time domains $[17,18,23]$. The skeleton is a good choice of marker since the spectrogram has a poor time-frequency resolution, but its components are located at the same position as that of the desired components that we wish to reconstruct from the WVD.

The method performs its operation as follows:

- The spectrogram of the input signal is computed, and the result is called $g_{i}(\mathbf{x})$.

- The noisy time-frequency transformation, a Wigner-Ville transform, is also computed from the input signal. The image corresponding to the distribution that displays interference is referred to as $f(\mathbf{x})$.

- The image $g_{i}(\mathbf{x})$ is pre-processed using a homotopy preserving skeleton algorithm [20], thus resulting in $g^{\text {skel }}(\mathbf{x})$. The method removes crosscomponents from $f(\mathbf{x})$ using $g^{\text {skel }}(\mathbf{x})$ as a marker for the $\lambda$-reconstruction operation. In comparison with the spectrogram, the output of the scheme the reconstructed image $f_{r}(\mathbf{x})$ - is an image with improved time-frequency resolution, without cross-components.

The selection of $\lambda$ plays an important role in improving the results. When we apply the proposed method to a diagram showing interference, the higher 


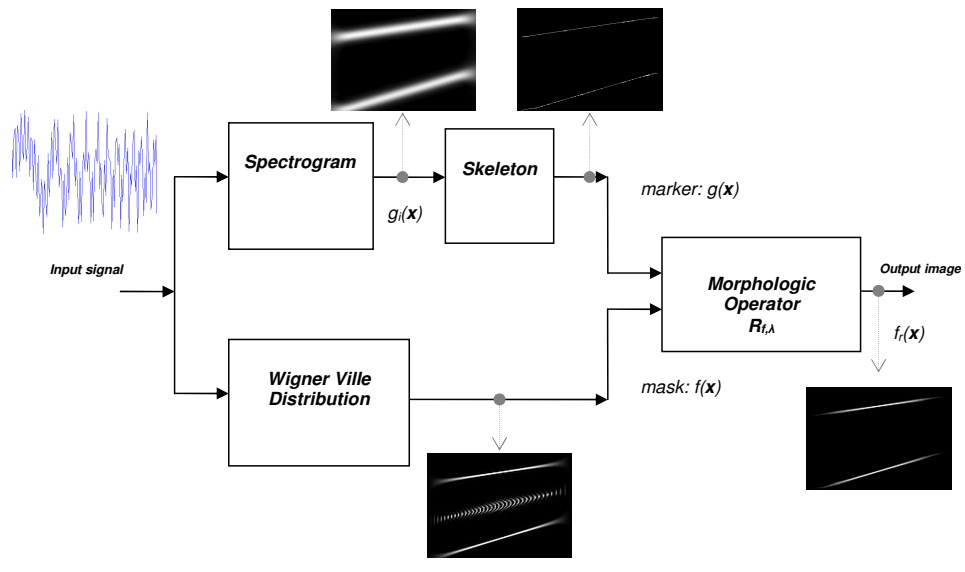

Figure 3: General scheme of the $\lambda$-RCCR method.

the value of $\lambda$, then the higher the value of interferences that can be reduced 
by the method. However, if $\lambda$ increases strongly we could also eliminate signal information. The selection of the accurate value of $\lambda$ is now discussed.

\subsection{Selection of parameter $\lambda$}

An error function has been formulated in order to evaluate the results found with our method for different values of $\lambda$. The $\lambda_{\text {optimum }}$ is the value of $\lambda$ that minimizes the error function given by Equation 2 .

As stated above, the skeleton of a signal contains the minimum quantity of information needed to locate the signal in both dimensions. In order to evaluate the error function, MSE minimization is employed. For every value of $\lambda$ we compute the difference in energy between the skeleton of the original signal, $g^{\text {skel }}(\mathbf{x})$, and the skeleton computed from the reconstructed diagram, $f_{r \lambda_{i}}^{s k e l}(\mathbf{x})$, using a value of $\lambda=\lambda_{i}$ (see Equation 2).

$$
\operatorname{error}\left(\lambda_{i}\right)=\frac{\left|\sum_{\mathbf{x}} g^{\text {skel }}(\mathbf{x})-\sum_{\mathbf{x}} f_{r \lambda_{i}}^{s k e l}(\mathbf{x})\right|^{2}}{\sum_{\mathbf{x}} f_{r}^{s k e l}(\mathbf{x})}
$$

When the diagram is reconstructed using $\lambda=\lambda_{\text {optimum }}$, the skeleton of the spectrogram and the skeleton corresponding to the reconstructed diagram are likely to be very similar. This is due to the fact that, when processing the WVD with the appropriate value of $\lambda$, the interference terms will disappear and as such do not contribute to the skeleton. In addition, the signal terms will remain in their original position contributing to the skeleton in the same way as the signal components from the spectrogram. When the WVD is reconstructed with a value of $\lambda$ lower than the $\lambda_{\text {optimum }}, \lambda<\lambda_{\text {optimum }}$, several interference components that touch the signal terms will remain following reconstruction. These unremoved components will contribute to the skeleton image, thereby increasing the value of the error function. On the other hand, when the WVD is reconstructed with a value of $\lambda$ higher than the $\lambda_{\text {optimum }}, \lambda>\lambda_{\text {optimum }}$, the cross-terms are removed and do not contribute to the skeleton of the reconstructed image. However, by using $\lambda>\lambda_{\text {optimum }}$, desired information of the signal, which disappears from the skeleton of the reconstructed diagram, will be lost, thus increasing the error function.

In Section 5.1, an algorithm for obtaining the $\lambda_{\text {optimum }}$ is discussed. The results obtained using the $\lambda$-RCCR method are also presented. The method operation has been compared with the methods proposed in $[12,13]$ and also with classical methods, such as the filtering using 2-D Gaussian kernels. Furthermore, the correct performance of the method when it is applied to other types of transforms has been demonstrated.

\section{Results and Discussion}

The operating method is applied to a set of synthetic test signals $x_{1}(n)$, $x_{2}(n)$, and $x_{3}(n)$, and to two real signals $x_{4}(n)$ and $x_{5}(n)$. 
To clarify the formulation of the synthetic signals let us introduce how to formulate the linear frequency-modulated and sinusoidal frequency-modulated signals.

On the one hand, with $f_{0}$ being the normalized initial frequency and $f_{1}$ the normalized final frequency, we can compute the $N$ points record of a linear frequency-modulated signal as:

$$
\begin{gathered}
f(n)=f_{0} \cdot\left(n-\frac{N}{2}\right)+\frac{f_{1}-f_{0}}{2(N-1)} \cdot\left[(n-1)^{2}-\left(\frac{N}{2}-1\right)^{2}\right], \quad n=1,2, \ldots, N \\
x(n)=\exp (j \cdot 2 \cdot \pi \cdot f(n))
\end{gathered}
$$

On the other hand, let us call $f_{0}$ the normalized initial frequency, $f_{1}$ the normalized final frequency and $N_{p}$ the sinusoidal period. We define the following parameter $\phi$,

$$
\phi=\operatorname{acos}\left(\left(1 / 4-\frac{f_{1}+f_{0}}{2}\right) \cdot \frac{2}{f_{1}-f_{0}}\right)
$$

We can compute the $N$ points record of a sinusoidal frequency-modulated signal as:

$$
\begin{gathered}
\Phi=\pi \cdot\left(f_{0}+f_{1}\right) \cdot n+\frac{f_{1}-f_{0}}{2} \cdot N_{p}\left(\sin \left(\frac{2 \pi \cdot n}{N_{p}}+\phi\right)-\sin (\phi)\right), \\
n=-N / 2+1,-N / 2+2, \ldots, N / 2 \\
x(n)=\exp (j \cdot \Phi)
\end{gathered}
$$

With this basic formulation, a brief outline of these signals is presented bellow:

- $x_{1}(n)$ : it is composed by two linear frequency-modulated signals (see Equation (3)) whose components are well-separated in the frequency domain. The parameters for the first component were fixed as: $f_{0}=0$ and $f_{1}=0.2$. The parameters for the second component were selected as: $f_{0}=0.35$ and $f_{1}=0.45$. For both components $N=128$ points were computed and $x_{1}(n)$ is obtained adding the two components.

- $x_{2}(n)$ : it is composed by two linear frequency-modulated signals (see Equation (3)) whose components are not well-separated in the frequency domain. The parameters for the first component were fixed as: $f_{0}=0$ and $f_{1}=0.4$. The parameters for the second component were selected as: $f_{0}=0.22$, and $f_{1}=0.5$. For both components $N=128$ points were computed and $x_{2}(n)$ is obtained adding the two components. 
- $x_{3}(n)$ : it is composed by two sinusoidal frequency-modulated signals (see Equation (4)) whose components are not well-separated in the frequency plane. The first sinusoid has the following parameters $f_{0}=0.25, f_{1}=0.35$, and $N_{p}=100$. The second sinusoid has parameters $f_{0}=0.35, f_{1}=0.45$, and $N_{p}=100$. For both components $N=128$ points were computed and $x_{3}(n)$ is obtained adding the two components.

- $x_{4}(n)$ and $x_{5}(n)$ : two underwater signals. The first signal is a real signal from a beluga whale and the second from a tugboat.

The automatic classification of these signals is a typical application of our developments.

The spectrogram and Wigner-Ville distributions of the discrete time signals presented hereafter were computed using the MATLAB time-frequency toolbox [24]. Being $N$ is the number of points of the signal under test, the spectrogram of this signal is computed for time instants from 1 to $N$, using $N$ frequency bins and a $\frac{N}{4}$-point Hamming analysis window normalized to unit energy. On the other hand, the Wigner-Ville distribution was computed for the same time instants and using the same number of frequency bins. In this paper, the effect of the type and the size of the analysis window used to compute the spectrogram is not considered since the skeleton of the spectrogram contains the minimum quantity of information to locate each component. Therefore, the skeleton will not change when different windows are used. Moreover, the skeleton will have the same geometry independently of the original resolution of the computed spectrogram.

\subsection{Estimation of parameter $\lambda$}

Before discussing the results obtained from the method, it is important to calculate the value of the selected $\lambda$. The method described in Section 4.1 has been used to numerically evaluate the error function (see Equation 2) using the reconstructed diagram of signal $x_{3}(n), x_{4}(n)$, and $x_{5}(n)$ for several values of $\lambda_{i}$ in the interval from 0 to 100. Remember that if the parameter $\lambda=0$ the $\lambda$-reconstruction operator performs the same operation as the reconstruction operator, and the interferences which touch the desired signals are not removed as was demonstrated in [16].

Figure 4 shows the error evolution for different values of $\lambda$. The "*" line corresponds to the evaluation of the error function for the WVD diagram of an underwater signal received from a tugboat, $x_{5}(n)$; the " $\square$ " line corresponds to the evaluation of the error function for the WV diagram of an underwater signal of the beluga whale, $x_{4}(n)$; the "o" line corresponds to the reconstruction of the WVD of the sinusoidal signal $x_{3}(n)$.

Moreover, several simulations over a varied set of signals, of a similar nature to the set herein presented, corroborate the earlier results. These simulations provide values of $\lambda_{\text {optimum }}$ near to 25 in all cases, and this value is in accordance with the value that we have empirically set as optimum. From this, we can assume and generalize the value of $\lambda_{\text {optimum }}=25$ as a representative choice for these kinds of signals. 


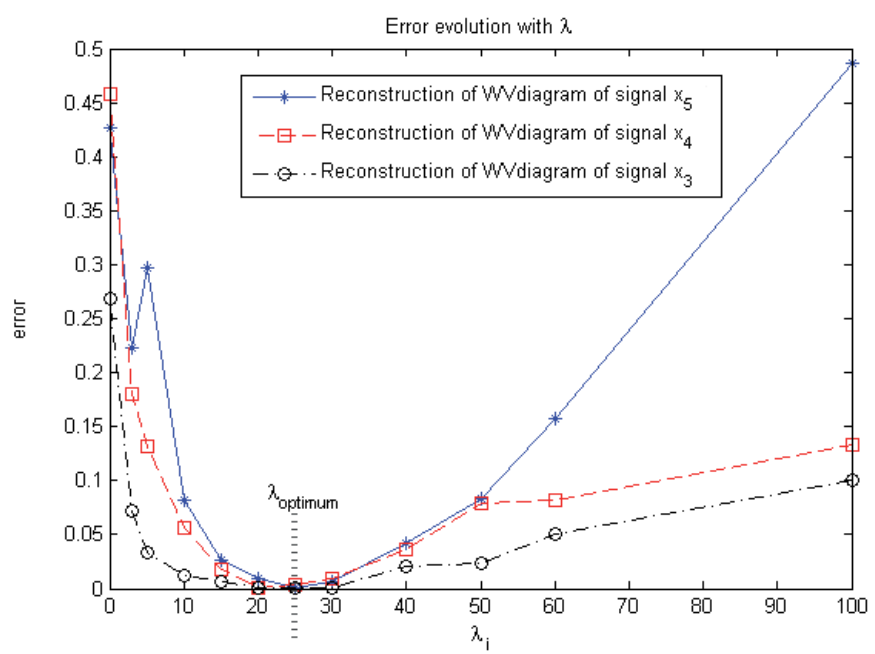

Figure 4: Evaluating the error function for different values of $\lambda$.

Next, we provide some representative examples applying the method over the set of test and real signals. Firstly, in section 5.2, we compare our method results with those obtained using the method proposed in $[12,13]$. Both methods are applied over the set of test signals. Secondly, in the same section, we have included a new example where total overlap between signal and interferences is managed; the results obtained with the proposed method are compared to those obtained with techniques of filtering using 2-D Gaussian kernels. Then, in section 5.3, the $\lambda$-RCCR method is tested over the set of real signals. Finally, in section 5.4, the method is also tested when applying it over other types of frequency distributions.

\subsection{Method Comparison}

The method proposed in [12] has been tested to compare their results with those obtained using our method. The former method reduces cross-components by combining the Fourier-Bessel (FB) expansion with the WVD. The FourierBessel expansion decomposes a multi-component signal into a number of monocomponent signals [25], and the WVD is applied over each mono-component signal. In the same way as proposed in [16], this is a powerful tool to eliminate interferences from the WVD when signal components are well-resolved. If interferences overlap or touch signal components, the method cannot be expected to correctly perform the separation of frequency components. Hence, the WVD will return a diagram showing interferences. The authors in [12] have recently presented an improvement to their method in order to solve this problem. This technique combines the time-order representation based on short-time FourierBessel expansion and the Wigner-Ville distribution (WVD) [13] which noticeably improves the results of the previous method in some cases, as we can 
corroborate in the examples.

We present three representative examples to discuss these points: Figures 5,6 , and 7 represent the results obtained when processing signals $x_{1}(n), x_{2}(n)$, and $x_{3}(n)$, respectively.

Figure 5 represents the results of the method proposed in [12] in comparison with the $\lambda$-RCCR method. Figures 6 and 7 represent the results of the method proposed in [12] and the results obtained with its improvement [13] in comparison with the $\lambda$-RCCR method.

In Figures 5.a, 6.a, and 7.a we represent the Fourier-Bessel coefficients of the three input signals, $x_{1}(n), x_{2}(n)$, and $x_{3}(n)$, respectively. Note how the two components from each signal can be correctly separated. Once the two main components from the signals are separated, we can retrieve each signal component separately and compute one WVD from every component. Figures 5.c and 5.d, 6.c and 6.d, and 7.c and 7.d show the WVD from each signal retrieved. Figure 5.e represents the sum of the two images 5.c and 5.d. In the same way, Figure 6.e represents the sum of the two images 6.c and 6.d and Figure 7.e represents the sum of the two images 7.c and 7.d. From Figure 5.e, we can emphasize the outstanding results of the method [12] applied to $x_{1}(n)$ whose components are well-separated in the frequency domain. On the other hand, when signal components are not well-separated (see Figures 6.e and 7.e), the method [12] was unable to achieve good results since it cannot correctly separate their Fourier-Bessel components. Figures 6 (f, g, and h) and 7 (f, g, and h) show the result obtained using the improved method [13]. Specifically, Figures 6 (f and $g$ ) and 7 ( $f$ and $g$ ) represent the WVD of each signal component, retrieved from $x_{2}(n)$ and $x_{3}(n)$, respectively, using the short-time FB expansion. Figure 6.h shows the sum of images 6.f and 6.g. In the same way, Figure 7.h shows the sum of images 7.f and 7.g. In Figure 6.h, we can observe how in the case of a linear frequency-modulated signal $\left(x_{2}(n)\right)$ the interferences are noticeably reduced using the improved method. In the case of non-linear modulated signals (Figure 7), the improvement achieves a good quality of the signal terms but it does not reduce the interferences.

Figures 5 (b, f, g, and h), 6 (b, i, j, and k), and 7 (b, i, j, and k) show the images used and obtained using the $\lambda$-RCCR method. In Figures 5.b, 6.b, and $7 . \mathrm{b}$ we have the spectrogram of the three input signals $x_{1}(n), x_{2}(n)$, and $x_{3}(n)$, that is $g_{i 1}(\mathbf{x}), g_{i 2}(\mathbf{x})$, and $g_{i 3}(\mathbf{x})$, respectively. Figures 5.f, 6.i, and 7.i represent the skeleton of images $g_{i 1}(\mathbf{x}), g_{i 2}(\mathbf{x})$, and $g_{i 3}(\mathbf{x})$, respectively, that is $g_{1}^{\text {skel }}(\mathbf{x}), g_{2}^{\text {skel }}(\mathbf{x})$ and $g_{3}^{\text {skel }}(\mathbf{x})$ (the markers). Figures 5.g, 6.j, and 7.j represent $f_{1}(\mathbf{x}), f_{2}(\mathbf{x})$, and $f_{3}(\mathbf{x})$, respectively, the reference images for the morphological operator, these being the images that correspond to the WVD of $x_{1}(n), x_{2}(n)$, and $x_{3}(n)$. Finally, figures $5 . \mathrm{h}, 6 . \mathrm{k}$, and $7 . \mathrm{k}$ represent the results of applying our method (using $\lambda=25$ ), which are the reconstructed images $f_{r}^{W V 1}(\mathbf{x}), f_{r}^{W V 2}(\mathbf{x})$, and $f_{r}^{W V 3}(\mathbf{x})$. The three reconstructed images contain only the desired information from the WVD, preserving time-frequency resolutions and avoiding any cross-components. We can highlight the satisfactory results obtained over the three test signals independently of the component locations, even when interferences touch signal terms. 


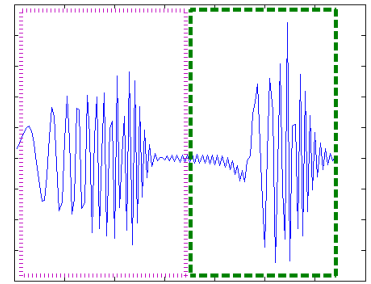

(a)

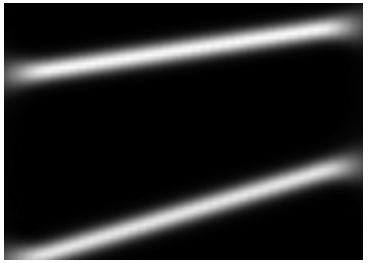

(b)

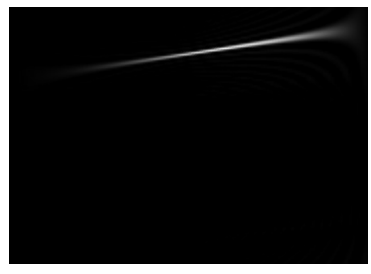

(c)

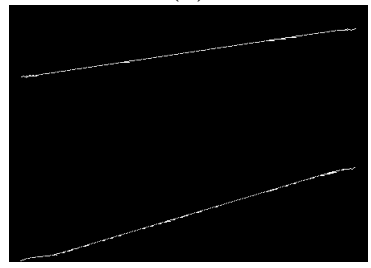

(f)

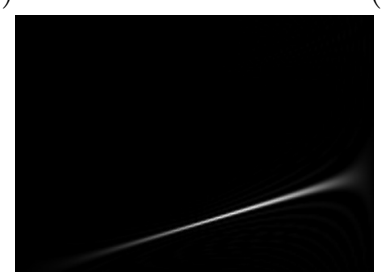

(d)

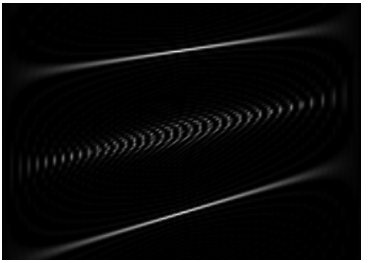

$(\mathrm{g})$

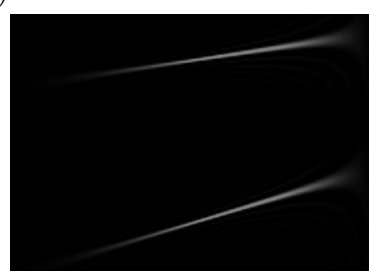

(e)

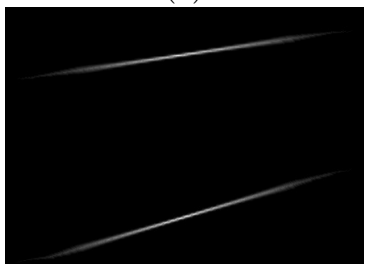

(h)

Figure 5: (a) $F B$ coefficients of $x_{1}(n)$; (b) Spectrogram of signal $x_{1}(n), g_{i 1}(\mathbf{x})$; (c) WVD of the retrieved signal 1; (d) WVD of the retrieved signal 2; (e) composition of images of figure c and figure d; (f) $g_{1}^{\text {skel }}(\mathbf{x}) ;(\mathrm{g}) \mathrm{WV}$ transform of $x_{1}(n), f_{1}(\mathbf{x}) ;(\mathrm{h})$ reconstructed image $f_{r}^{W V 1}(\mathbf{x})$.

Finally, we illustrate the operation of our method in comparison with classical techniques. We show an experiment where total overlap between signal and interference components is tested.

Specifically, we now compare the $\lambda$-RCCR results with those obtained using WVD 2-D Gaussian kernels. As stated in Section 2, many research papers have been published on this topic with the selection of the correct kernel being the main drawback of these techniques since it depends of the type of signal.

In the following example, we use a three component signal composed by three harmonically related chirps (instantaneous frequencies of f, $2 \mathrm{f}$, and $3 \mathrm{f}$ ). Here the cross-terms between the first and third component will land on top of the second component so as to test the ability of our algorithm to manage complete overlapping components. Also, filtering with 2-D Gaussian kernels is recognized to work well with problems of this kind.

The tested chirp signal is composed by three linear frequency-modulated signals. The first frequency-modulated component is a discrete-time signal with $N=256$ samples, having initial normalized frequency of $f_{0}^{1}=0.01$ and final 


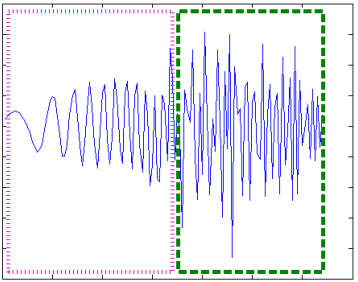

(a)

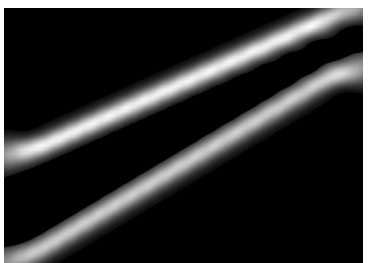

(b)

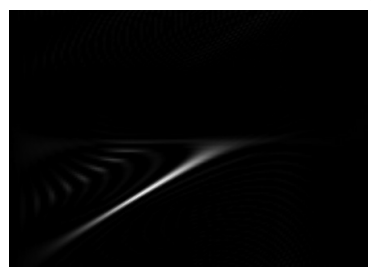

(c)

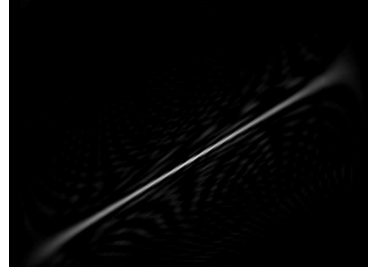

(f)

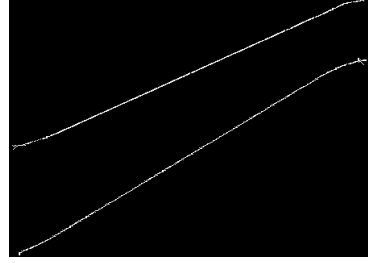

(i)

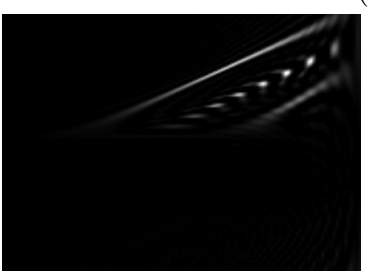

(d)

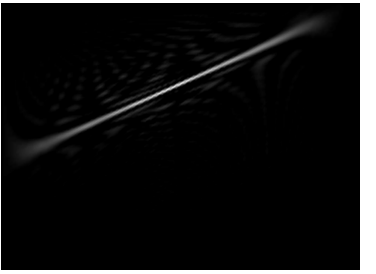

(g)

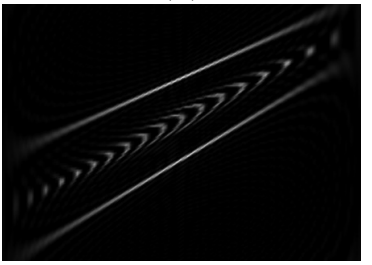

(j)

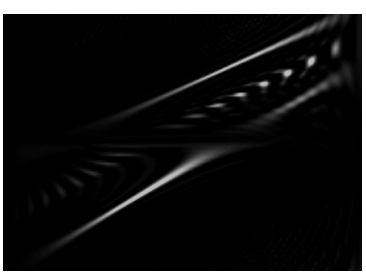

(e)

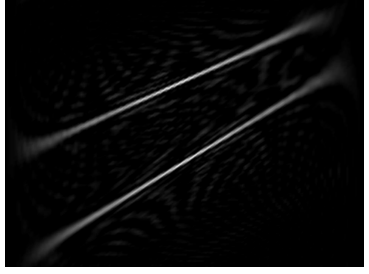

(h)

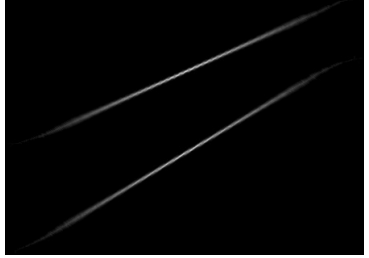

(k)

Figure 6: (a) FB coefficients of $x_{2}(n)$; (b) SP of signal $x_{2}(n), g_{i 2}(\mathbf{x})$; (c) WVD of the retrieved signal 1 using FB coefficients; (d) WVD of the retrieved signal 2 using FB coefficients; (e) composition of images of figure $\mathrm{c}$ and figure d; (f) WVD of the retrieved signal 1 using shorttime FB coefficients; (g) WVD of the retrieved signal 2 using short-time FB coefficients; (h) composition of images of figure f and figure g; (i) marker $g_{1}^{\text {skel }}(\mathbf{x})$; (j) WV transform of $x_{2}(n)$, $f_{2}(\mathbf{x}) ;(\mathrm{k}) f_{r}^{W V^{2}}(\mathbf{x})$.

normalized frequency $f_{1}^{1}=0.1$. The second component is a signal with the same number of points, which have initial normalized frequency of $f_{0}^{2}=2 f_{0}^{1}$ and final normalized frequency of $f_{1}^{2}=2 f_{1}^{1}$. Finally, the third linear frequencymodulated signal contains the same number of points and has initial normalized frequency of $f_{0}^{3}=3 f_{0}^{1}$ and final normalized frequency of $f_{1}^{3}=3 f_{1}^{1}$. The three components of the signal have a time reference for the phase of $\frac{N}{2}$.

Figure 8 shows the results of filtering the three-component chirp signal using 


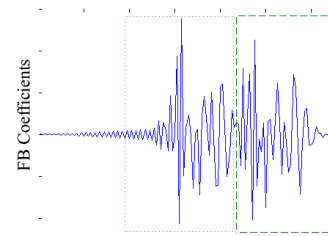

Order

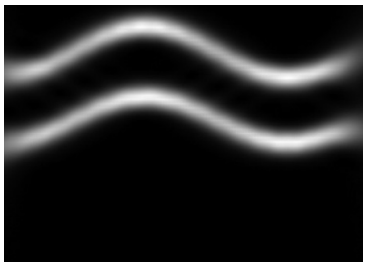

(b)

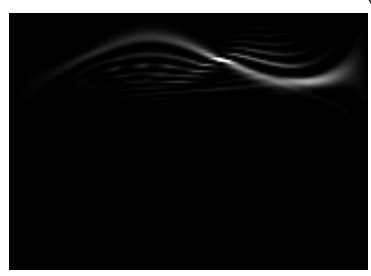

(c)

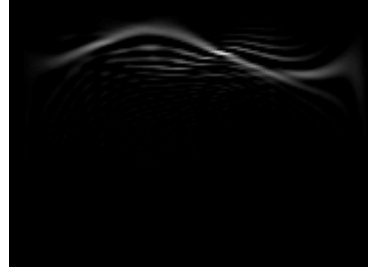

(f)

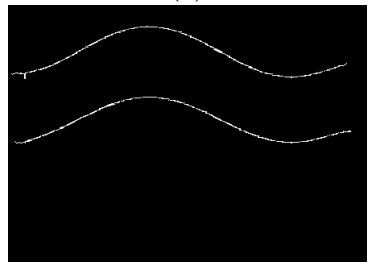

(i)

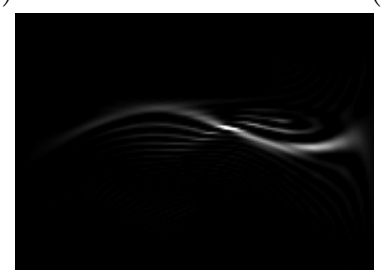

(d)

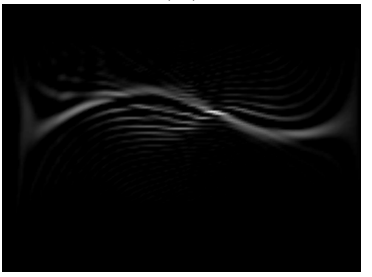

(g)

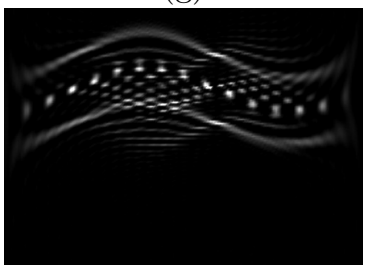

(j)

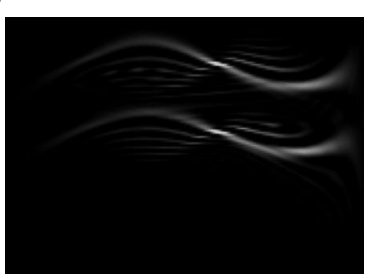

(e)

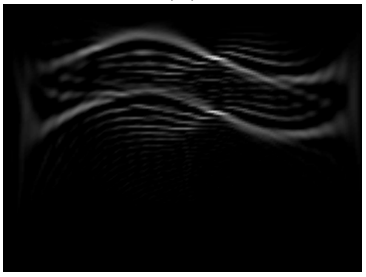

(h)

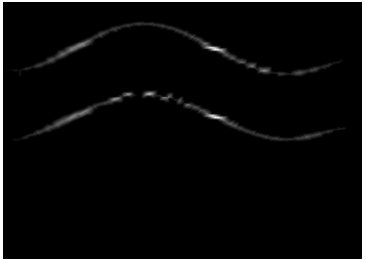

(k)

Figure 7: (a) $F B$ coefficients of $x_{3}(n)$; (b) SP of $x_{3}(n)$; (c) WVD of the retrieved component 1 using FB coefficients; (d) WVD of the retrieved component 2 using FB coefficients; (e) composition of images of figure b and figure c; (f) WVD of the retrieved component 1 using short-time FB coefficients; (g) WVD of the retrieved component 2 using short-time FB coefficients; (h) composition of images of figure $\mathrm{f}$ and figure g; (i) marker $g_{3}(\mathbf{x})$; (j) WV transform of signal $x_{3}(n) ;(\mathrm{k}) f_{r}^{W V 3}(\mathbf{x})$.

a 2-D Gaussian kernel, with a temporal smoothing window that we call $\mathrm{G}$ and a frequency smoothing window that we call H. Figures 8.a and 8.b show the spectrogram and Wigner-Ville distributions of the signal, respectively. Figure 8.c shows the results of filtering using a 63-point time smoothing Gaussian window and a 63 -point frequency smoothing Gaussian window. Figure 8.d shows the results using 127 -point $\mathrm{G}$ window and 63 -point $\mathrm{H}$ window. Finally, Figure 8.c shows the results of filtering using a 63 -point time smoothing Gaussian window 


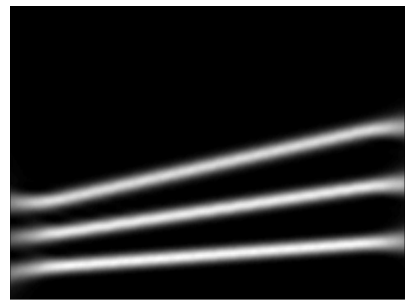

(a)

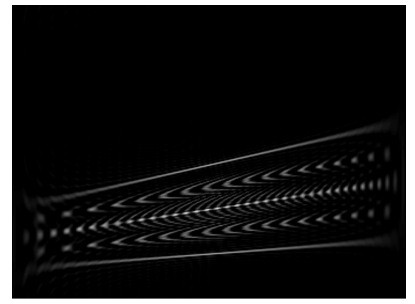

(b)

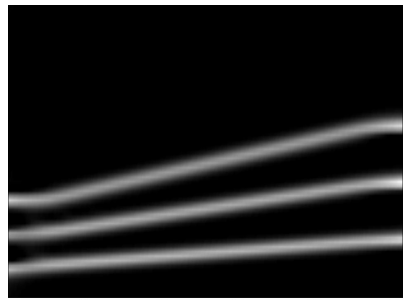

(c)

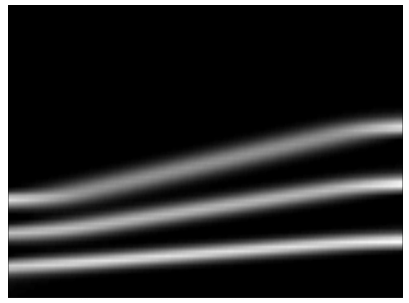

(d)

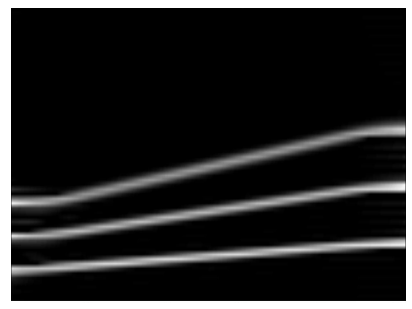

(e)

Figure 8: (a) Spectrogram of the three-component chirp signal. (b) WVD of the threecomponent chirp signal. (c) Result of the filtering using 63-point $\mathrm{G}$ window and 63-point $\mathrm{H}$ window. (d) Result of the filtering using 127-point $\mathrm{G}$ window and 63-point $\mathrm{H}$ window. (e) Result of the filtering using 63-point $\mathrm{G}$ window and 127 -point $\mathrm{H}$ window.

and a 127-point frequency smoothing Gaussian window.

Figure 9 shows the results of applying the $\lambda$-RCCR method to the same three-component chirp signal. Figure 9.a shows the marker for the morphological operation, and Figure 9.b shows the reconstructed image.

Considering the results shown in Figures 8 and 9, we can conclude that the 2-D filtering techniques remove interference terms but reduce the WVD resolution. As the length of the windows increase, the resolution also increase, but it never reaches the WVD resolution. The $\lambda$-RCCR method achieves a high resolution of components located at $\mathrm{f}$ and $3 \mathrm{f}$ frequencies removing the interferences. The drawback is that the component locates at frequency $2 \mathrm{f}$ (the case of total overlap) can not be completely recovered using the $\lambda$-RCCR method. Other morphological operators can be used to improve this situation, and this research will be done in future work.

\subsection{Application To Real Signals}

In this section, we discuss the operating method applied to two underwater signals: a signal from a beluga whale, $x_{4}(n)$, and a signal from a tubboat, $x_{5}(n)$.

Figures 10.a and 11.a are the spectrogram of $x_{4}(n)$ and $x_{5}(n)$, respectively. Figures 10.b and 11.b are the skeletons of the spectrogram that are used as markers, $g_{4}^{\text {skel }}(\mathbf{x})$ and $g_{5}^{\text {skel }}(\mathbf{x})$. Figures 10.c and 11.c represent the WVD of $x_{4}(n)$ and $x_{5}(n)$, respectively. The reconstructed images, $f_{r}^{W V 4}(\mathbf{x})$ and $f_{r}^{W V 5}(\mathbf{x})$, are represented in Figures 10.d and 11.d, respectively. 


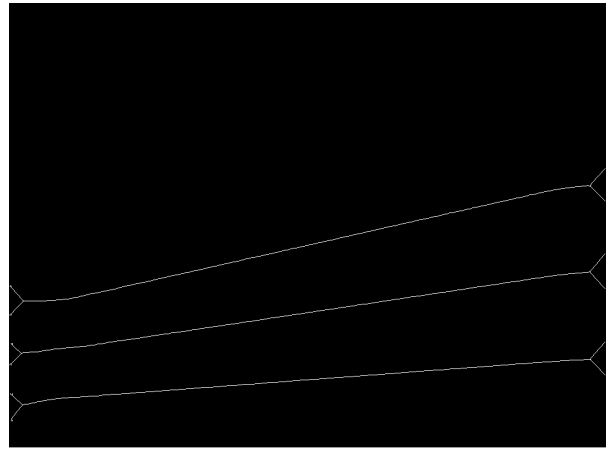

(a)

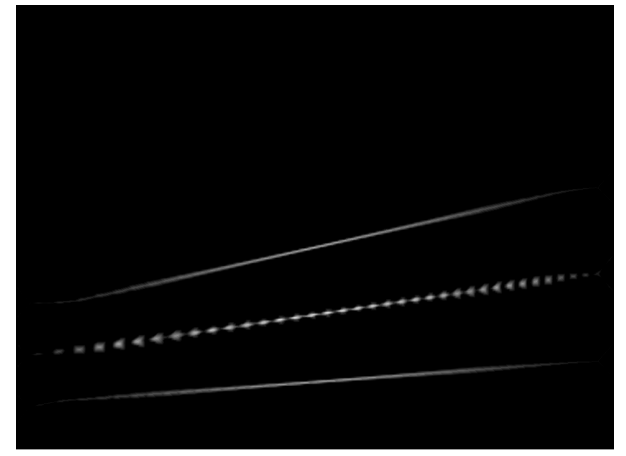

(b)

Figure 9: Results of applying our method to a three-component chirp signal. (a) marker ; (b) reconstructed image.

From here, we can conclude that our method achieves great results when it is applied over real signals. Moreover, Figure 11.d not only highlights the satisfactory results of the method over real signals, it also demonstrates the usefulness of the $\lambda$-reconstruction in comparison with the reconstruction operator $(\lambda$-reconstruction with $\lambda=0)$. In [16], the same signal, $x_{5}(n)$, was used to illustrate the drawback of the reconstruction operator when it is used to reconstruct diagrams whose interference terms overlap the desired signal.

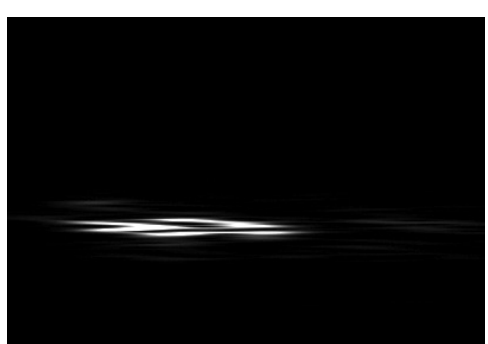

(a)

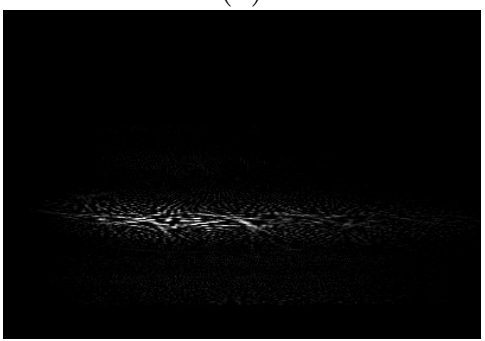

(c)

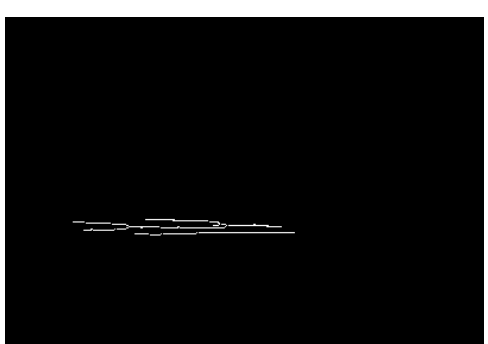

(b)

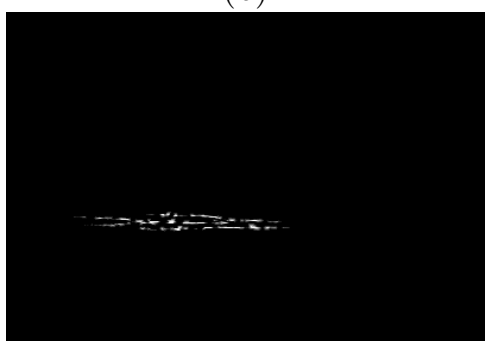

(d)

Figure 10: (a) Spectrogram of signal $x_{4}(n)$; (b) marker $g_{4}^{\text {skel }}(\mathbf{x})$; (c) WVD of $x_{4}(n)$; (d) $f_{r}^{W V 4}(\mathbf{x})$. 


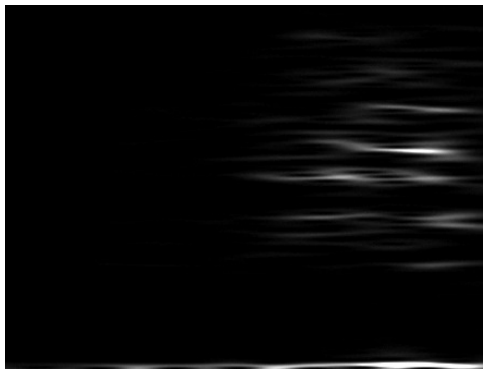

(a)

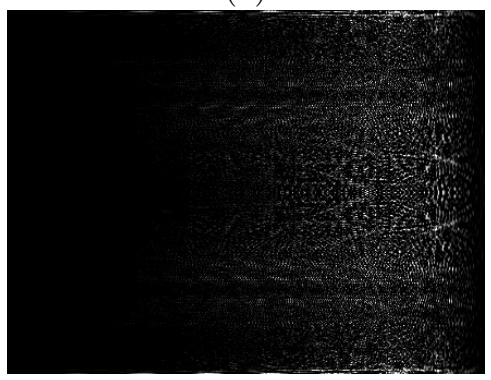

(c)

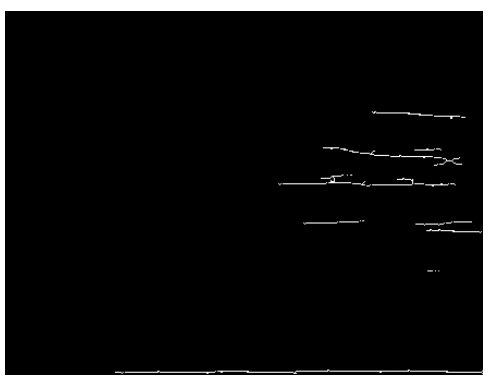

(b)

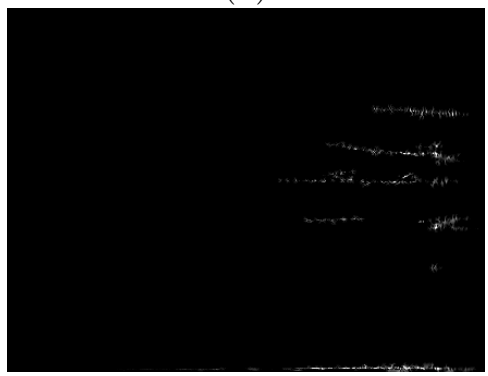

(d)

Figure 11: (a) Spectrogram of $x_{5}(n)$; (b) marker $g_{5}^{\text {skel }}(\mathbf{x})$; (c) WVD of $x_{5}(n)$; (d) reconstructed image $f_{r}^{W V 5}(\mathbf{x})$.

\subsection{Using Other Time-Frequency Distributions}

In this section, the results of applying the propossed method to other timefrequency distributions are shown, namely: the Margenau-Hill (MH) and the Choi-Williams (CW) distributions. Both transforms have different interference geometries in comparison with the WVD [1].

Once again, in this example, the input signals are $x_{3}(n)$ and $x_{4}(n)$. As such, the images used as markers for the $\lambda$-reconstruction operation are the same as those used in the previous subsections, mainly images $g_{3}^{\text {skel }}(\mathbf{x})$ (Figure 7.b) and $g_{4}^{\text {skel }}(\mathbf{x})$ (Figure 10.b).

Figures 12.a and 13.a display the images corresponding to the Choi-Williams transform of both input signals, respectively. These images have been used as references for the $\lambda$-reconstruction operator. Figures 12.b and 13.b represent $f_{r}^{C W 1}(\mathbf{x})$ and $f_{r}^{C W 2}(\mathbf{x})$, which are the reconstructed images from 12.a and 13.a using the proposed method.

In the same way, Figures 12.c and 13.c represent the images that correspond to the Margenau-Hill transform of signals $x_{3}(n)$ and $x_{4}(n)$ and are used as references. Figures 12.d and 13.d represent $f_{r}^{M H 1}(\mathbf{x})$ and $f_{r}^{M H 2}(\mathbf{x})$, the reconstructed images.

The correct operation of the method and its application to other transforms, that have different interference geometries, is corroborated by Figures 12.b, 12.d, 13.b, and 13.d. It can be observed how the method strongly reduces the cross- 
components, thereby preserving time-frequency resolutions.

To conclude, we point out that our method achieves great results when applied over real signals regardless of the interference geometry and regardless of the type of transform applied.

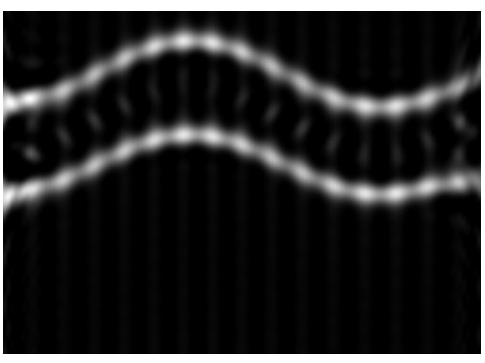

(a)

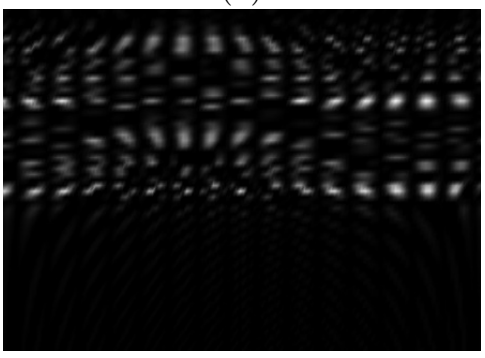

(c)

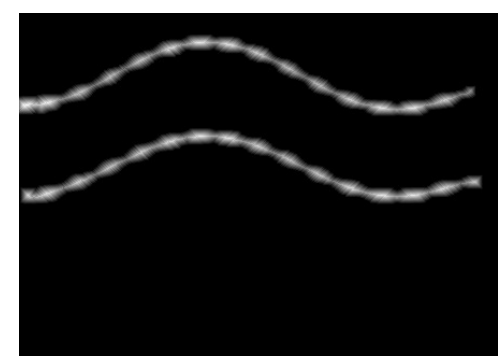

(b)

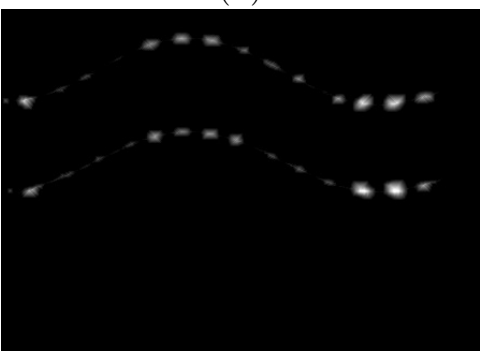

(d)

Figure 12: (a) CW distribution of signal $x_{3}(n)$; (b) reconstructed image $f_{r}^{C W 1}(\mathbf{x})$; (c) $\mathrm{MH}$ distribution of signal $x_{3}(n)$; (d) reconstructed image $f_{r}^{M{ }^{11}}(\mathbf{x})$.

\section{Conclusions}

A new method for the removal of cross-components from time-frequency transformations has been presented. The proposed method uses morphological operators to reduce interference components from time-frequency images. It has been tested not only over test signals, but also over real examples of underwater signals. In comparison with other techniques in the same field, our method overcomes the main drawback, providing a correct operation even when signal and interference terms overlap. Moreover, it can be applied to distributions with different interference geometry, and it preserves time-frequency resolutions.

The method achieves outstanding results in removing interference components not only from the WVD, but also from other types of transforms such as the Margenau-Hill (MH) or the Choi-Williams (CW) distributions. Its great performance when it is applied over transforms with different interference geometries has also been demonstrated. 


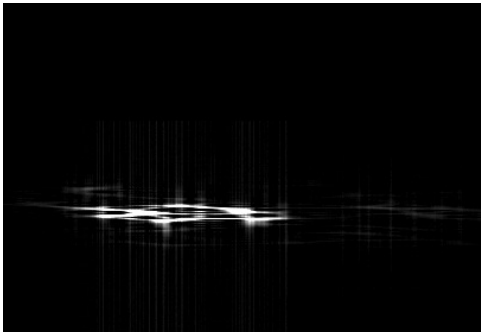

(a)

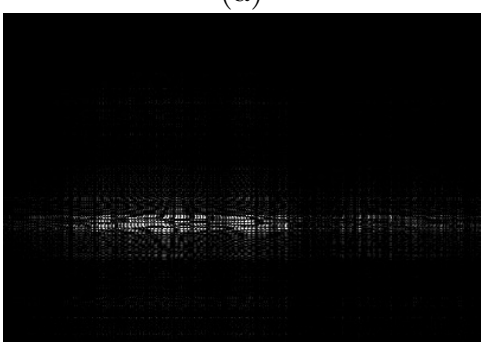

(c)

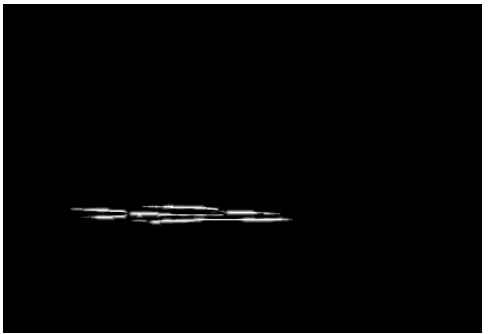

(b)

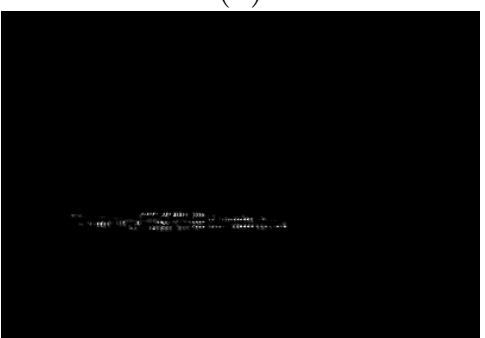

(d)

Figure 13: (a) CW transform of $x_{4}(n)$; (b) $f_{r}^{C W 2}(\mathbf{x})$, (c) MH transform of $x_{4}(n)$; (d) $f_{r}^{M H 2}(\mathbf{x})$

\section{References}

[1] L. Cohen, Time-frequency distributions-a review, in: Proceedings of the IEEE, Vol. 77, 1989, pp. 941-981.

[2] T. Claasen, W. Meclenbrauker, The wigner distribution-a tool for time frequency signal analysis-part 2: Discrete time signals, Phillips Journal of Research 35 (1980) 276-300.

[3] B. Boashash, Time-Frequency Signal Analysis and Processing A Comprehensive Reference, Oxford, Elsevier Science, 2003.

[4] P. Flandrin, Temps-fréquence, Traitement du Signal, Hermes, Trait des Nouvelles Technologies, 1993.

[5] F. Hlawatsch, G. Boudreaux-Bartels, Linear and quadratic time-frequency signals representations, IEEE Signal Processing Magazine 9 (1992) 21-87.

[6] F. Hlawatsch, P. Flandrin, The interference structure of the wigner distribution and related time-frequency signals representations, Wigner Distribution -Theory and applications in Signal Processing, Amsterdam, The Netherlands: Elsevier 35 (1997) 372-389.

[7] P. Flandrin, Some features of time-frequency representations of multicomponent signals, in: Proceedings of the IEEE Int. Conf. Acoust., Speech, Sig. Proc., 1984, pp. $266-269$. 
[8] A. Jansen, T. Claasen, On positivity of time-frequency distributions, in: Proceedings of the IEEE Trans. Acoust., Speech, Sig. Proc., Vol. 33, 1985, pp. 1029-1032.

[9] M. E. Tagluk, M. J. English, The analysis of ecg signals using timefrequency techniques, in: Proceedings of the 19th Annual International Conference of the IEEE on Engineering in Medicine and Biology Society, Vol. 3, 1997, pp. 1320-1323.

[10] R. G. Baraniuk, D. L. Jones, A radial gaussian, signal dependent timefrequency representation, Signal Proc. 32 (1993) 263-284.

[11] D. L. Jones, R. G. Baraniuk, An adaptive optimal-kernel time-frequency representation, IEEE Transactions on Signal Processing 43 (1995) 23612371.

[12] R. B. Pachori, P. Sircar, A new technique to reduce cross terms in the wigner distribution, Digital Signal Processing 17 (2) (2007) 466-474.

[13] R. Pachori, P. Sircar, Time-frequency analysis using time-order representation and wigner distribution, in: Proceedings of the IEEE Tencon Conference, Hyderabad, India, 2008.

[14] D. Jones, R. G. Baraniuk, Elimination of cross-components of the discrete pseudo wigner distribution via image processing, in: Proceedings of the IEEE Int. Conf. Acoust., Speech, Sig. Proc., 1989, pp. 2231-2233.

[15] L. Tao, Y. Shao-Quan, Improvement on joint time-frequency representation with application of image processing technique, in: Proceedings of the Fifth International Conference on Computational Intelligence and Multimedia Applications, 2003, pp. 243-248.

[16] S. Gómez, V. Naranjo, R. Miralles, An application of morphologic filters to remove non-linear distorsions from time-frequency representations of acoustic signals, in: Proceedings of the 19th International Congress on Acoustic, Madrid, 2007.

[17] J. Serra, Image analysis and mathematical morphology, Academic Press, 1988.

[18] J. Serra, Image analysis and mathematical morphology vol II. Theoretical advances, Academic Press, 1988.

[19] J. Serra, P. Salembier, Connected operators and pyramids, in: Proccedings of SPIE. Image Algebra and Mathematical Morphology, 1993.

[20] P. Soille, Morphological Image Analysis:Principles and Applications. Second Edition, Springer, 2002. 
[21] F. Meyer, Alpha-beta flat zones, levelings and flattenings, in: Proceedings of the 6th international symposium on mathematical morphology, 2002, pp. $47-68$.

[22] V. Naranjo, A. Albiol, J. Mossi, A. Albiol, Morphological lambdareconstruction applied to restoration of blotches in old films, in: Proceedings of the The 4th IASTED International Conference on Visualisation, Imaging and Image Processing, 2004.

[23] L. Ji, J. Piper, Fast homotopy-preserving skeletons using mathematical morphology, IEEE Transactions on Pattern Analysis and Machine Intelligence 14 (6) (1992) 653-664.

[24] F. Auger, P. Flandrin, P. Gonçalvès, O. Lemoine, Timefrequency toolbox, CNRS (France) Rice University-USA, 1995-1996.

[25] R. B. Pachori, P. Sircar, Speech analysis using fourier-bessel expansion and discrete energy separation algorithm, Digital Signal Processing Workshop, 12th - Signal Processing Education Workshop 4 (2006) 423-428. 